\title{
Le solennel et le parcimonieux dans l'alimentation. Les pratiques gastronomiques comme source de distinction des élites brésiliennes
}

The solemn and the parsimonious of food and eating among Brazilian elites: considerations in discourses of gastronomy and culinary traditions Lo solemne y lo parcimonioso : consideraciones sobre el discurso gastronómico y las tradiciones de cocina de la élite brasileña

\section{Carolina PULICI}

\section{OpenEdition} Journals

Édition électronique

URL : https://journals.openedition.org/ideas/441

DOI : 10.4000/ideas.441

ISSN : 1950-5701

\section{Éditeur}

Institut des Amériques

\section{Référence électronique}

Carolina PULICI, « Le solennel et le parcimonieux dans l'alimentation. Les pratiques gastronomiques comme source de distinction des élites brésiliennes », IdeAs [En ligne], 3 | 2012, mis en ligne le 03 janvier 2013, consulté le 19 octobre 2022. URL : http://journals.openedition.org/ideas/441 ; DOI : https://doi.org/10.4000/ideas.441

Ce document a été généré automatiquement le 19 octobre 2022.

Creative Commons - Attribution - Pas d'Utilisation Commerciale - Pas de Modification 4.0 International - CC BY-NC-ND 4.0

https://creativecommons.org/licenses/by-nc-nd/4.0/ 


\title{
Le solennel et le parcimonieux dans l'alimentation. Les pratiques gastronomiques comme source de distinction des élites brésiliennes
}

\author{
The solemn and the parsimonious of food and eating among Brazilian elites: \\ considerations in discourses of gastronomy and culinary traditions \\ Lo solemne y lo parcimonioso : consideraciones sobre el discurso gastronómico y \\ las tradiciones de cocina de la élite brasileña
}

\section{Carolina PULICI}

\section{Introduction}

1 L'alimentation est une pratique socialement distinctive, comme le montrent des études récentes attestant de la persistance des différences sociales en matière de consommation alimentaire (Régnier et al., 2006). En ce qui concerne les pratiques gastronomiques des élites d'un pays comme le Brésil, ancienne colonie portugaise et une des dernières nations à abolir l'esclavage (1888), les travaux contemporains soutiennent que les bourgeoisies locales n'ont jamais voulu se confondre avec les peuples indigènes, et ont préféré adopter une identité européenne. Ainsi, même la feijoada, devenue un symbole de l'identité nationale au $\mathrm{XX}^{\mathrm{e}}$ siècle ${ }^{1}$, resterait un plat surtout populaire, " marginal dans les habitudes alimentaires des élites » (Dória, 2009 : $32)^{2}$.

2 Les enjeux de distinction liés à l'alimentation ont été un thème cher aux chercheurs en sciences sociales qui ont noté comment les pratiques et les visions à l'égard de la nourriture constituent une dimension oubliée de la lutte des classes. Étudiant le régime alimentaire du peuple au cours de la révolution industrielle, E. P. Thompson souligne que le prestige social des aliments est aussi crucial que leurs qualités diététiques. Même 
si les nutritionnistes nous vantent aujourd'hui les propriétés de la pomme de terre, dit l'auteur, ce produit était associé aux immigrants Irlandais et, de ce fait, son introduction à la place du pain fut ressentie comme une "conspiration" visant à réduire les pauvres Anglais au niveau des Irlandais (Thompson, 1988 : 287). Rejetant l'économisme qui conçoit la stratification sociale uniquement comme un ensemble de positions socioéconomiques, Richard Hoggart s'attache au style de vie des classes populaires anglaises où «la bonne table » est avant tout «un repas copieux plutôt qu'un menu équilibré » (Hoggart, 1957 : 70). Également convaincu qu'une classe se définit par sa consommation autant que par sa position dans les rapports de production, Pierre Bourdieu constate qu'à l'éthique de la sobriété pour la minceur revendiquée par les dominants («sachant se dominer»), les dominés opposent « une morale de la bonne vie » qui consiste à bien manger et à bien boire et à savoir entrer dans une relation familière supprimant les distances (Bourdieu, 1979). Plus récemment, Faustine Régnier et al. ont aussi discuté le souci de la minceur comme symbole d'excellence corporelle, en ayant remarqué que, pour les groupes aisés, l'alimentation est plus que jamais un élément de distinction «qui permet de faire preuve de son bon goût, de ses talents, le tout assorti d'un intense souci pour le corps » (Régnier et al., 2006 :3).

3 La recherche présentée ici partage cet intérêt de lire une classe à travers l'alimentation et se propose donc de prolonger ces travaux. Elle postule que, dans les classes supérieures brésiliennes, les pratiques alimentaires constituent un mode de distinction sociale qui conduit à valoriser le repas parcimonieux et investi d'une certaine solennité. À partir d'une enquête sur le style de vie d'une fraction des élites de São Paulo, la capitale économique du pays, on analysera les discours sur "quoi », "combien » et «comment» manger et leur rôle dans la légitimation de modes de savoir-vivre socialement discriminants. Si le corps bien maîtrisé (et la morale gastronomique qui l'accompagne) est devenu un modèle d'excellence, le but de cet article est de voir comment ceux qui peuvent le mieux s'adapter à cette nouvelle forme de " présentation de soi » revendiquent leur supériorité sur ceux qui résistent à la transformation de leur rapport à la nourriture. 


\section{Méthodologie de l'enquête}

Cette enquête est issue d'une recherche plus vaste qui utilise du matériel de presse et des traités de savoir-vivre, réalise des entretiens et mène une analyse secondaire de statistiques. Pour ce qui concerne l'univers de la cuisine, notre réflexion repose sur deux types de sources.

D'une part, on a recueilli des guides d'étiquette récemment publiés et des critiques gastronomiques parues dans la presse pauliste entre 2005 et 2009. La constitution de ce corpus empirique a priorisé les ouvrages les mieux placés dans les listes de vente et les quotidiens aux tirages les plus importants et adressés à un public favorisé.

D'autre part, trente entretiens semi-directifs ont été réalisés avec des représentants des classes supérieures de São Paulo. Comme il serait impossible de discuter, dans le cadre de cet article, les conditions dans lesquelles s'est développé le travail de terrain avec des groupes fortunés qui imposent de fortes résistances au contact et tendent à dominer la situation de l'entretien, nous expliciterons seulement de façon synthétique les principes pris en compte pour constituer notre population. Comme il n'existe pas d'équivalent du «Bottin mondain » ou du Who's Who, on a repéré des agents socialement désignés comme «riches » en faisant intervenir des critères "réputationnels » issus des récits publiés dans la presse et de notre cercle de rapports professionnels. Face à l'impossibilité d'introduire des données de structure, comme les rendements d'opérations financières et des valeurs de patrimoine (Medeiros, 2005), on s'est encore servi de critères institutionnels en s'adressant à des occupants de positions de pouvoir reconnues mais également de paramètres concernant l'appropriation privilégiée de l'espace urbain. Les difficultés d'approcher les hauts cercles et leur faible délimitation empirique ont imposé une flexibilité de sélection des informateurs incompatible avec les exigences de constitution d'un échantillon et, en ce sens, notre analyse est d'abord exploratoire ${ }^{3}$.

On s'attachera d'abord à décrire certaines normes qui s'imposent aujourd'hui comme distinctives en matière d'alimentation et de manières de table. Dans un deuxième temps, on se centrera sur la façon dont des fractions des classes aisées paulistes réagissent à ces appels d'esthétisation de la cuisine et sur les visions que leurs membres construisent d'eux-mêmes et des autres à partir de leurs attitudes alimentaires déclarées, lesquelles contribuent aussi à former la « réalité » de ces élites.

\section{L'art de bien consommer et de se tenir à table}

Norbert Elias a établi, dans ses différents ouvrages, que l'adhésion à un style de vie plus raffiné constitue une forme de contrôle de soi qui augmente les chances, pour un groupe supérieur, d'affirmer et de maintenir son pouvoir. Dans La civilisation des mœurs, il s'intéresse aux ouvrages de "civilité " de la Renaissance qui témoigneraient des grands changements dans le comportement de l'homme européen, alors plus contraint à se soumettre à des normes de convivialité précises. En ce qui concerne l'art de bien se tenir à table, un traité fameux de ce temps avertit à de nombreuses reprises qu'il est impératif de savoir éviter la voracité. Ainsi, s'il ne faut pas se précipiter, pour reprendre Érasme cité par Elias (1973: 96), « le premier sur le plat qu'on apporte » car «c'est le propre des paysans de plonger les doigts dans la sauce ». Les personnes qui 
«dévorent plus qu'elles ne mangent » et celles qui «émettent des grognements de porcs » sont également mal vues (ibid. : 131).

6 Aussi surprenant que cela puisse paraître, on constate qu'une bonne partie des recommandations présentées dans les manuels d'étiquette parus au long des années 1990-2000 au Brésil suivent encore de près quelques prescriptions formulées par Érasme il y a cinq siècles. Parmi les règles de conduite adressées à celui qui cherche à s'imposer sur ceux qui restent prisonniers des sentiments " naturels ", l'alimentation demeure un domaine crucial: «Les gens ne sont pas invités pour être nourris, mais pour contribuer en quelque sorte au succès d'une rencontre. Par leur charme, leur beauté, leur intelligence, leur capacité à être drôles. Brillez dans l'exercice de votre partie [...]. Moins est souvent plus dans les fêtes et les dîners. Manger moins. Boire moins » (Leão, 2007 : 34-35). S'accordant avec la vision selon laquelle un dominant doit toujours exhiber une bonne maîtrise de son corps (Pinçon et Pinçon-Charlot, 2000 : 91), le manuel prescrit encore: «[...] ne vous jetez pas sur l'assiette comme si vous étiez en train de sortir d'une grève de la faim. Si vous êtes vraiment un Pantagruel, mangez un petit quelque chose avant de partir de chez vous » (ibid. : 109). Pendant un vol aérien, «mangez peu. Buvez peu [...]. Ne vous comportez pas du genre 'je veux tout ce à quoi j'ai droit'. Ne vous précipitez pas sur le champagne, sur le caviar » (ibid. : 129)4.

7 L'apologie du contrôle des satisfactions corporelles apparait dans un autre guide contemporain, écrit par une spécialiste en étiquette qui affirme avoir été choquée de voir, lors d'un séjour dans un resort, des gens qui se précipitaient sur le buffet ${ }^{5}$ : « C'était laid à voir: de vrais gloutons en train de mettre des pyramides de nourriture devant eux et de dévorer les aliments [...]. Un spectacle de voracité qui se répétait à chaque repas. Le fait d'avoir devant soi plusieurs options - sans avoir à payer à la carte ou au poids ne justifie pas ce non-sens grossier et excessif. Contrôler les impulsions et les appétits, c'est ce qui distingue les êtres civilisés des bêtes sauvages »(Kalil, $2007: 150)^{6}$. Un autre manuel - intitulé Culture et élégance - mentionne encore le "show d'horreur d'assiettes qui débordent » et donne ainsi à voir à quel point le laisser-aller à table déclasse: « C'est dans les petits détails qu'on découvre qui est qui. Dans un restaurant avec buffet à volonté, par exemple, évitez de gorger l'assiette avec des aliments et des assaisonnements de différentes origines qui ne vont pas bien ensemble. Soyez tranquille [...]. Reprendre n'est pas inélégant, mais faire une assiette immense montre de la voracité et c'est extrêmement laid» (Leão, $2005: 212)^{7}$.

8 Si l'appétit vorace est l'attribut de ceux qui ne se détournent pas du plaisir « facile » des besoins corporels, l'alimentation parcimonieuse est, a contrario, réservée à ceux qui, plus libérés des urgences du monde, sont aussi plus aptes à se régaler avec l'art culinaire "cérébral» qui s'impose comme distinctif ${ }^{8}$. Le numéro que la revue du quotidien Folha de São Paulo a consacré au "Dîner du siècle » est indicatif de cette nouvelle morale gastronomique qui glorifie la nourriture de « l'esprit » au détriment de celle $\mathrm{du}$ "corps ». Cette rencontre réunissant seize grands chefs espagnols a reçu un public prêt à payer environ 2300 euros et à goûter « de petits morceaux de l'avantgarde espagnole», comme le carpaccio de pastèque cuit pendant neuf heures. L'intention de faire de l'art de manger un des beaux-arts éclate dans les analogies entre l'alimentation et le monde artistique: «Dans une certaine mesure, c'est comme si on rassemblait Picasso, Goya et Miró pour peindre chacun son tableau [...]. De petits morceaux de l'avant-garde espagnole bien là, à la portée des quatre-vingt invités. Mais de petits morceaux vraiment petits. La cuisine contemporaine n'aime pas la 
gloutonnerie. 'Ce n'est pas une nourriture ordinaire, mais quelque chose qui nous fait penser', dit Ricardo Castilho, directeur éditorial de la revue Plaisirs de la table, essayant d'expliquer la déconstruction à la Dalí faite par Adrià et son groupe » (Bastos, 2008 : $11)^{10}$.

Selon un grand chef, restaurateur et critique gastronomique, "l'héritage de cette 'nouvelle-nouvelle' est en train de réaffirmer les petits plats. Qui supporte encore, actuellement, de manger une assiette de docker pleine à ras bords? » (Horta, 2008 : E5). Et «combien» manger, la quantité, n'est pas la seule cible des propagateurs du "gastronomiquement correct ", qui prescrivent aussi « comment» on doit se nourrir. D'un côté le propriétaire de quelques-uns des restaurants les plus sophistiqués du pays déplore le fait que «le Brésilien avait l'habitude du plat unique, qui n'est pas accepté dans la haute gastronomie ", ayant admis avoir déjà refusé " une demande de côtelette à la milanaise avec spaghetti aux fruits de mer sur la même assiette " (Bergamasco, 2007 : C6). De l'autre, un manuel d'étiquette exhorte son lecteur à être « super exigeant à table » car il s'agirait « du niveau de civilisation et de raffinement » (Leão, 2007 : 154).

\section{Un « bien de famille »}

10 À en juger par les récits que nous avons pu recueillir, les "vieilles familles " sont, même dans un pays du «nouveau monde » comme le Brésil, surreprésentées dans ce domaine des bonnes et belles manières, faisant de l'art de bien se tenir à table un de leurs traits distinctifs. La chronique «Expertes en recevoir", parue dans une revue idéal-typique du goût bourgeois, indique que ces techniques de la sociabilité mondaine sont censées faire partie d'un apprentissage précoce, puisque le «pedigree social » est l'atout qui semble attester, en fin de compte, l'authenticité du savoir-faire « des plus grandes hôtes » locaux: "Filles des familles les plus traditionnelles de la ville, ces expertes dans l'art de recevoir ont commencé à apprendre le b.a.-ba de l'étiquette au berceau. Et ont renforcé leur éducation dans des collèges renommés. Et se sont mariées à des hommes prospères et bien introduits " (Alonso, 2003) ${ }^{11}$. Cette conversion d'un mode particulier d'acquisition de la culture en manière légitime (et presque « instinctive») d'exister est courante sur les marchés mondains, qui tendent à exiger des savoirs moins susceptibles d'être acquis à l'école et plus liés simplement à une «bonne » naissance ${ }^{12}$.

11 La suprématie de ceux pour qui l'élégance à table est un «bien de famille » est tellement reconnue dans ces univers qu'un ouvrage de civilité n'hésite pas à recommander le mensonge si besoin est. Si, au cours d'un dîner, quelqu'un pose une question sur l'origine de l'argenterie et de la porcelaine, il faut dire: "'elles appartenaient à ma mère'. C'est très chic, cela ajoute une touche de noblesse (même si ce n'est pas vrai)» (Leão, $2007: 33$ ). On voit ici se matérialiser la vision goffmanienne du bluff comme principe même de toute vie sociale (Goffman, 1973 : 76). Une vision naturaliste des pratiques gastronomiques se donne à voir encore dans une chronique sur un des quartiers les plus bourgeois de la ville, où il s'agirait, pour un connaisseur de son " code ", d'une « hérésie de consulter les menus de certains restaurants " (Nunes, 2005). Le mépris pour l'individu récemment enrichi est à vrai dire banal dans ces mondes "raffinés ", comme si tout argent gagné en dehors de l'héritage faisait preuve d'arrivisme. Lors d'une interview à la Folha de São Paulo, le responsable des affaires du Château Pichon Longueville Comtesse de Lalande répond ainsi à la question «Les 
consommateurs de vin connaissent-ils vraiment le vin? » : Au Brésil, le marché est partagé. Il y a les connaisseurs, qui appartiennent aux familles les plus anciennes, et les nouveaux milliardaires, amateurs, qui achètent plutôt pour étaler. Ils sont comme des vitrines parce que, de fait, ils consomment le vin et l'exhibent aux amis » (11/09/2008, p. E2). C'est ainsi qu'un guide du savoir-vivre interdit la moindre référence au capital économique nécessaire à l'art de bien consommer: «Si vous avez invité quelqu'un au restaurant, ne faites jamais, mais vraiment jamais, un quelconque commentaire sur le prix d'un plat. Même pas pour dire qu'il est très abordable, et moins encore pour dire qu'il est cher. Si vous voulez être réellement élégant, dites tout de suite: «Vous ne voulez pas du homard ?'» (Leão, 2007 : 67). Discutant les modes légitimes, pour un couple, de se faire des cadeaux, le manuel L'amant élégant: un guide d'étiquette à deux avertit : "Offrir du champagne est toujours élégant, mais veillez à ce qu'il soit d'une marque indubitablement supérieure» (Matarazzo, 2001 : 68) ${ }^{13}$. Retraduction symbolique d'une place sociale privilégiée, cette «stylisation de la vie » dont parlait Weber $(2000: 183)$ est donc forcément une revendication de pouvoir sur ceux qui restent dominés par des urgences ordinaires.

$\mathrm{Si}$ les discours qui figurent dans des manuels d'étiquette et des critiques gastronomiques peuvent ne pas dire grand-chose sur la réalité objective des pratiques, ils mettent toutefois en évidence certaines normes d'excellence sociale et aussi les relations non négligeables entre prestige et consommation alimentaire. En tant que critères de savoir-vivre investis de légitimité, ces préceptes ne peuvent pas être autonomisés de l'ensemble du style de vie dans lequel ils se réalisent le plus pleinement. Ils doivent, de ce fait, être envisagés dans le cadre des luttes symboliques opposant les différents groupes engagés dans la représentation du monde social et, plus spécifiquement ici, dans l'imposition de principes admissibles de rapport à l'alimentation.

\section{Maîtrise du corps et rejet de la voracité populaire}

Discutant les limites des études utilisant seulement des méthodes déclaratives, JeanPierre Poulain signale que les réponses spontanées tendent à restituer les normes intériorisées et que, du coup, de telles données "ne peuvent pas être considérées comme des variables comportementales objectives» (Poulain, 2002:66). Ayant mené notre recherche principalement au moyen d'entretiens semi-directifs, on note que les enquêtés peuvent en effet donner une «impression idéalisée " (Goffman, 1973 : 40) d'eux-mêmes, en tout cas plus conforme à la représentation qu'ils veulent fournir de leur groupe. Même s'ils évoquent la consommation quotidienne de nourritures ordinaires (comme le riz aux haricots rouges), la plupart d'entre eux s'applique à projeter une image de grande familiarité avec des plats qui bénéficient d'un prestige social supérieur, comme ceux qui sont plus nettement associés à la gastronomie française et italienne $\mathrm{e}^{14}$. Si, accompagnant « la vogue du terroir» (Régnier et al., 2006 : 83), ils peuvent valoriser une certaine cuisine réputée caractéristique du Brésil ${ }^{15}$, leur alimentation se distingue par le choix supplémentaire de produits marqués symboliquement par rapport aux ingrédients courants (comme du homard, du merlan, des escargots, du caviar, du veau, des crevettes, des artichauts, du foie gras, du saumon $^{16}$, du filet mignon, du rosbif, des calamars, des asperges et du riz au canard). 

à la «table copieuse $»^{17}$, nos informateurs condamnent le «franc-manger » associé aux plus démunis: «La classe sociale la plus simple mange basiquement avec des couverts, je crois, [...] et fait une grande assiette. C'est une montagne qui est sur l'assiette, et tout sera mangé » (banquier et philanthrope). Dans ce contexte de rejet de la gloutonnerie, le «peuple» figure comme prisonnier de ses sensations, comme en témoigne cette description archétypique de l'alimentation populaire au Brésil:

« Du riz, des haricots, de la viande rôtie, de la farofa [accompagnement à base de farine de manioc torréfiée], cette farofa avec du manioc cuit, c'est vraiment une assiette de docker [...]. Il y a un restaurant à très bon prix, note 5,5 sur 10 . On y va pour bavarder, manger, parce que c'est près du travail. Mais parce qu'il est abordable, on voit venir des gens... conducteurs de taxi, des gens plus simples. C'est fou de voir ce qu'ils mangent, comment c'est possible? Comme c'est bon marché, il faut manger pour toute la semaine, vous voyez? » (Cadre supérieur d'un important groupe d'ingénierie).

Les autoreprésentations avec lesquelles les interviewés cherchent à créer ou à maintenir des différences par rapport aux autres segments sociaux expriment l'éloge du sacrifice des appétits immédiats au bénéfice des récompenses à venir, comme la beauté et la santé. En ce sens, les classes les moins favorisées seraient les plus imperméables à des valeurs qui risqueraient de rendre désuètes leurs attitudes traditionnelles par rapport à la nourriture. Le témoignage d'une "grande dame ", psychanalyste et épouse d'un industriel, révèle à quel point le goût est un indice de l'idée que chaque classe a du corps (et des effets de l'alimentation sur le corps):

«Je crois que tout le monde fait un régime. Mais bon, le peuple mange beaucoup. Par exemple, ici à la maison je dépense plus d'argent avec la nourriture des domestiques qu'avec la mienne, vous voyez ? D'accord, ça se comprend, ils font plus de travail physique. Encore que je fais aussi beaucoup de travail physique [référence aux deux heures et demie de gymnastique quotidienne]. Mais je ne m'empiffre pas $»^{18}$.

'imaginaire des distinctions de classe insurmontables marque aussi le récit d'un grand chef, restaurateur et propriétaire de restaurants dans le domaine de la haute gastronomie, quand il nous parle du cas où il a reçu une commande pour faire un dîner offert à tous les employés d'une entreprise, depuis le président jusqu'aux occupants des postes les plus modestes. Vu que ce genre de repas est rare - pour ne pas dire inexistant - pour les individus dépourvus de toutes les formes de capital, ces derniers ne se sont pas retenus face à cet événement vécu comme extraordinaire:

"J'ai donc servi de la dinde rôtie, un couscous ${ }^{19}$ et je ne sais pas quoi, coucous aux crevettes, n'est-ce pas ? Et alors Dona Luísa [qui travaille pour lui] y est allée et elle a dit en revenant: «Je suis pauvre, mais je vais vous dire, avec les pauvres c'est difficile, mon Dieu, ils ont attaqué la nourriture!' c'est parce qu'elle est habituée à aller dans des buffets de gens... plutôt élégants, n'est-ce pas, où on mange à peine [...]. On m'a raconté que quand la deuxième dinde est arrivée, les gens ont applaudi, vous savez?».

Emblème du groupe, l'alimentation parcimonieuse et faite de plats qui ne font pas grossir est évoquée par tous les enquêtés, à l'opposé du stéréotype toujours présent du "peuple» fasciné par les repas plus nourrissants : «[Je mange] plus de poisson. J'achète de la viande rouge pour le personnel, sinon...» (collectionneuse d'art, psychanalyste et ex-femme d'un industriel). L'absence de modération des classes populaires apparaît aussi dans le discours d'une plasticienne et veuve d'un industriel : «Ce qu'il y a dans la gamelle - je l'ai observé -, ils le mangent. Ça peut être énorme, 
mais ils mangent tout [...]. Je vois qu'ils mangent trop, une chose horrible». L'intempérance est l'attribut des pauvres aussi selon un chef : "On voit les gens faire des assiettes tellement grandes. Je veux dire, les gens simples font de très grandes assiettes [...]. Et les gens chics mangent peu ». Cette vision que la voracité est un invariant de l'alimentation populaire se dégage encore des commentaires de l'épouse d'un industriel: «Comme $90 \%$ de la population brésilienne est pauvre, on mange trop [...]. Peut-être parce que ici j'ai... [en faisant référence aux 5 domestiques], c'est une chose alarmante [...]. Ils mangent trop de tout, l'assiette est d'une taille! Et, par exemple, si vous alliez dans un restaurant d'un niveau plus, n'est-ce pas, populaire, c'est absurde ce qu'ils mangent ». Pour la femme d'un ministre à plusieurs reprises, les pauvres seraient aussi plus inclinés à l'excès alimentaire, sauf pour ceux qui sont en contact avec les plus riches, qui manifesteraient déjà un souci de manger plus correctement. Même un musicien qui, sur d'autres sujets, se montre détaché du goût bourgeois éprouve du dégoût face à ceux qui ne savent pas se contrôler face à la nourriture, attitude qu'il associe plutôt aux États-Unis où " $40 \%$ de la population » serait obèse ${ }^{20}$.

« Savez-vous ce que j'aime aussi ? C'est la honte... de la saucisse grillée ! »

Même si on ne vit plus dans un monde où des privilèges honorifiques accordaient à certains groupes l'exclusivité sur des aliments spécifiques et malgré le fait que, de plus en plus, c'est la façon de manger les mêmes nourritures qui donne lieu aux distinctions les plus importantes, il est intéressant de noter l'inertie sociale de certains aliments. Le sentiment de honte culturelle lié au goût pour les saucisses, par exemple, figure même dans les commentaires d'une artiste habitant une maison d'architecture moderne et, donc, moins inclinée en principe aux " goûts de tradition" (Bourdieu, 1979 : 301) associés aux bourgeois. Les propriétés déshonorantes de cette nourriture éclatent dans le cas de cette informatrice, car le fait d'être autrichienne et d'avoir grandi dans un pays connu pour la qualité et la diversité de ses saucisses ne suffit pas pour qu'elle se détache de l'image sociale de cet aliment dont elle fait une "consommation secrète ». Par un effet de "noblesse oblige », ses goûts alimentaires affichés ont donc été moins déterminés par les divisions ethniques que par les oppositions basées sur la distribution du capital.

Si on suit l'analyse d'Elias sur le processus de civilisation en Occident, on voit comment la classification des choses suit de près la classification des hommes : dans le traité de civilité d'Érasme, le fabricant de saucisse figure comme un des porteurs de mauvaises manières : «Le paysan se mouche dans sa casquette ou dans sa vareuse, le charcutier dans le creux du coude » (Érasme cité par Elias, 1973 : 94).

Concevant négativement le goût populaire comme goût lourd, nos enquêtés refusent les aliments dits grossiers et choisissent les produits à faible teneur en calories (« dans la vie quotidienne c'est juste une petite grillade pour ne pas grossir»), se mettent constamment au régime, arrêtent de dîner et de manger du pain au petit-déjeuner, remplacent les gâteaux par des fruits au dessert en adhérant, en somme, à une restriction délibérée qui est aux antipodes de l'art de boire et de manger sans réticence ( JJe m'interdis vraiment»; «Je blague en disant que chez moi il y a un 'réfrigérateur de pauvre' »). Dans l'ensemble des pratiques culturelles de cette population, la maitrise du corps est évoquée à plusieurs reprises et notamment quand il s'agit de discuter de 
l'alimentation: «Ce qui est vraiment bien - qui est un sage conseil et qu'on n'arrive pas à suivre toujours - c'est de se lever de table en pouvant manger encore, mais sans le faire » (banquier et philanthrope). L'ascétisme bourgeois semble alors être bien tel que le décrit Béatrix Le Wita (1988: 83), c'est-à-dire « une méthode de conduite visant à surmonter 'le status naturae', à soustraire l'homme à la puissance des instincts, à le libérer de sa dépendance à l'égard du monde et de la nature ».

\section{Esprit de solennité et attachement aux « tâches de représentation »}

19 Le rejet de la voracité populaire renvoie à une vision plus large selon laquelle le «peuple » ne ferait pas preuve de retenue d'une manière générale - et pas seulement à table -, ce qui ne prédispose pas à une autre attitude chère aux élites : les échanges cérémoniels d'une vie en société. Fidèles aux "tâches de représentation ", les détenteurs d'un goût "bourgeois » tiennent à esthétiser leur cuisine et leurs manières de table et, ainsi, les habitudes des classes défavorisées sont dévalorisées aussi en raison d'une indifférence supposée aux dimensions esthétiques de l'acte alimentaire: " Dans un restaurant plus pauvre vous voyez rarement un plat bien présenté » (grand entrepreneur de travaux publiques et philanthrope). Si la censure des besoins «naturels » est au principe de la disposition esthétique légitime (Bourdieu, 1979), il n'est pas difficile de comprendre pourquoi on attribue aux pauvres le penchant à la gloutonnerie mais également le manque de sens esthétique : «Je leur apprends [aux femmes de ménage] à ranger les feuilles de laitue [...] mais ça ne marche pas, et après ça me fatigue un peu parce qu'elles savent à peine parler [...]. Mais j'ai beaucoup fait pour cela parce que le visuel est très important pour moi » (collectionneuse d'art et exfemme d'un industriel). Une psychanalyste, «grande dame » et épouse d'un industriel, se plaint pour les mêmes raisons et dit que, contrairement à d'autres pièces utilitaires de la maison où il y a toujours des tableaux et des fleurs, la cuisine n'a rien de décoratif car les domestiques seraient indifférentes aux «mises en scène " de la vie quotidienne ${ }^{21}$.

20 La fidélité aux obligations de représentation se manifeste à plusieurs reprises en ce qui concerne l'apparence des repas: «Je trouve qu'une assiette mal présentée perd beaucoup [de son attrait] » (femme d'un important économiste et ministre à plusieurs reprises). Rentier et philanthrope, un enquêté a offert à son majordome un livre de Salvador Dalí avec les photos des plats et de la vaisselle de deux restaurants parisiens, le Lasserre et le Maxime, pour qu'il puisse s'en inspirer. Le parti de la stylisation de la vie apparaît aussi quand une héritière, psychanalyste et propriétaire d'une exploitation agricole, dit que «le plat, pour moi, c'est la table [...], il m'arrive de ne pas manger si c'est laid $»^{22}$. Dans son « penthouse » duplex, un chef et restaurateur entretient, sur la terrasse avec cheminée, des arbres fruitiers et un grenadier " qui est plutôt décoratif ». Il attache des orchidées dans les arbres des rues de ses résidences et de ses restaurants et cet engagement dans les tâches de représentation l'amène à conclure qu'en matière de cuisine, les barrières entre les classes s'élèvent moins en raison de la saveur et de l'assaisonnement que de " la manière de présenter, les tasses, comment on sert, tout ".

21 À la question "Croyez-vous qu'il y ait des différences dans les façons dont les différentes classes sociales préparent les aliments et les manières de les servir? ?, un collectionneur d'art, médecin propriétaire de clinique et héritier d'un propriétaire 
d'hôpital, estime qu'en banlieue les restaurants violent les normes culinaires consacrées (nourriture trop salée et trop grasse) et que cela se voit d'abord par leur architecture, attestant qu'en matière d'alimentation la pertinence d'une «mise en scène » n'est pas sans importance. Il considère que «la cuisine riche est la nouvelle cuisine » et admet avoir séjourné dans un Relais et Châteaux, près de Bordeaux, où il a payé 1000 dollars par jour pour déguster des repas en plusieurs étapes, c'est-à-dire pour pratiquer un rapport à l'alimentation qui s'oppose à l'immédiat (au bénéfice du différé) et à la fonction (en faveur de la forme). Dans sa maison entourée d'arbres fruitiers ("vous voyez du vert à partir de n'importe quel endroit») dans un quartier très exclusif de demeures dispersées dans une végétation abondante en pleine métropole, les repas sont solennisés et ne servent pas seulement à tuer la faim : «Je suis un homme ritualiste, je pense qu'être à table peut être un moment de réflexion [...]. Une phrase peut être très importante, donc, plus de rituel, plus de temps, vous comprenez ? [...] Il est apprécie, donc, d'avoir de bons couverts, un bon verre de vin ». $\mathrm{Si}$, comme nous avons vu auparavant, l'habitude du plat unique déshonore, le modèle de repas glorifié par la haute gastronomie ennoblit : invité à un dîner en sept étapes offert par un banquier et collectionneur dans un des restaurants les plus distingués de la ville, il observe : «Je ne pense pas du tout que cela soit bête [...]. Vous ne pouvez pas nier la Culture, la Culture, c'est de la connaissance cumulée !».

\section{Des aliments qui font l'honneur et le prestige de la gastronomie française}

« Du poisson, des crevettes, du homard, quoi que ce soit, ils arrivent très frais. Et alors je les fais descendre [dans la cuisine], elle [la cuisinière] sait déjà [comment faire], elle a été formée par ma fille qui est allée en Europe, qui a étudié l'art culinaire pendant cinq ans [...], elle a travaillé dans la cuisine d'Alain Senderens, place de la Madeleine, comment s'appelle ce restaurant? Il s'appelle Lucas Carton ».

Rentier, investisseur financier et philanthrope.

La négation des fonctions primaires de l'alimentation en vue de la transformation du repas en une cérémonie apparait dans le récit d'un banquier appartenant à d'anciennes familles. Recevant dans sa propriété de campagne les grands chefs Gaston Lenôtre et Roger Verger, l'enquêté organise un déjeuner destiné à leur faire une exposition de la cuisine brésilienne. Au cours de l'entretien, il nous montre le menu écrit en français et nous parle de ses discussions avec les illustres personnages de la « nouvelle cuisine » en France ("J'ai été très proche de Bocuse, de Verger »). Prolongeant, d'une certaine manière, le «racisme de classe » des manuels d'étiquette contre l'individu récemment enrichi, il fait référence à un banquier nouveau dans le métier, « un type de la zone nord de Rio [banlieue] » et qui possède aujourd'hui deux milliards de dollars, habite au Jardim Europa [ghetto des fortunés, où il habite aussi] et «boit du Magnum MoutonRothschild je ne sais pas comment». Ces groupes habitués à la "solennisation de l'existence " semblent avoir du mal à admettre sans réserve le parvenu, comme l'indique aussi le récit d'une ambassadrice et critique de décoration issue de familles traditionnelles. Au long de l'entretien, elle reconnaît avoir eu de la chance d'avoir appris les «bonnes manières " tout simplement à partir d'une insertion précoce dans un monde de personnes et de pratiques "civilisées" et a du mal à comprendre l'acquisition de ces savoirs en dehors du cadre familial, comme le révèle son étonnement à la découverte de l'existence de cours d'étiquette adressés à des publics 
différents : «Mais qu'est-ce que les gens apprennent ? Comment se tenir à la table ?» Les enjeux de distinction liés à l'alimentation s'expriment encore dans le récit d'un chef qui, ayant appris les bonnes manières au sein de la famille, désapprouve les clients récemment enrichis qui commandent ses services de restauration: "On fait de la dégustation et on voit que le type ne sait pas manger à table ».

La solennisation des pratiques gastronomiques se révèle encore dans tous les formalismes imposés à l'appétit immédiat. Une décoratrice et philanthrope issue des "vieilles familles" affirme avoir déjà introduit, dans les déjeuners hebdomadaires qu'elle offre à ses petits-enfants, le rituel aristocratique de l'eau parfumée à la lavande pour qu'ils puissent se laver les mains. L'art de se tenir à table est par ailleurs un composant essentiel de l'éducation des enfants : «Je me rappelle m'être renseigné sur comment mes enfants se comportaient à table» (banquier et philanthrope). L'insensibilité à ces observances cérémonielles lui déplaît profondément, comme l'indique ce souvenir d'une situation vécue dans un restaurant à Rome : " Il y avait à côté une personne qui mangeait la bouche ouverte, en faisant un tel bruit [...] que je ne pouvais plus manger! Alors là franchement. Pourquoi cela me dérangeait autant ? [...] Parce que c'est absolument désagréable ».

Avoir de bonnes manières de table n'est pas seulement estimé : c'est un indice de l'ancienneté de l'appartenance aux élites. Pour un rentier et philanthrope, c'est à table qu'on voit «si quelqu'un est bien élevé ou pas ». Le postulat qui fait de l'étiquette à table un indicateur de l'origine sociale émerge dans le récit d'un informateur qui a vécu l'expérience de la migration de classe. Ayant découvert l'infériorité sociale de ses parents à mesure qu'il quittait son monde d'origine, cet ancien président d'une entreprise multinationale et propriétaire d'une fabrique d'équipements hospitaliers avoue qu'une des choses qui lui ont fait comprendre que sa belle-famille appartenait à des couches sociales plus élevées a été le décalage entre la façon dont sa mère se tenait à table et les manières plus raffinées de sa future belle-mère qui était professeur. En illustrant «l'hypothèse selon laquelle la proximité sociale favorise les rapprochements affectifs alors que la distance sociale perturbe les liens affectifs » (de Gaulejac, 1987 : 84), il affirme avoir été étonné que sa femme ait pu s'intéresser à lui à ce stade de sa vie, quand il n'avait pas encore été « poli » culturellement ${ }^{23}$.

\section{Conclusion}

Pour incomplet qu'il soit, cet examen des habitudes alimentaires de certaines fractions des élites brésiliennes nous amène à conclure, tout d'abord, que les inégalités sont toujours importantes dans le domaine de la cuisine et des manières de table, surtout dans un pays comme le Brésil, dont l'augmentation générale du niveau de vie - et la position récemment acquise de sixième puissance économique mondiale - ne l'empêche pas de figurer parmi les nations présentant les plus grands indices de disparité sociale. Dans la ville la plus riche du seul pays lusophone de l'Amérique Latine, l'alimentation apparaît comme un des signes distinctifs des catégories aisées, qui affirment leur distance à l'égard du commun à travers l'esthétisation de leurs pratiques gastronomiques et la retenue de leur appétit alimentaire. La valorisation des repas investis de beauté visuelle et sensibles à des observances cérémonielles suggère encore que les grands bourgeois se trouvent parmi ceux qui résistent le mieux à ce qu'on a 
appelé «la dissolution des conventions de la consommation alimentaire » (GarciaGarza, 2010 : 439).

L'ascétisme électif revendiqué par des membres des classes supérieures est à la source de profits symboliques non négligeables et semble avoir comme contrepartie nécessaire la dépréciation du laxisme attribué aux couches populaires. Alors que le «peuple " ne résisterait pas aux impulsions alimentaires, l'élite fait preuve de maîtrise de soi et affiche l'adhésion au plaisir différé dont on jouit tout au long d'une vie, et pas seulement de façon immédiate. De telles représentations d'eux-mêmes et des autres témoignent de la persistance des enjeux de distinction liés à l'alimentation. Ainsi, même si l'on admet que le goût n'est pas réductible à l'appartenance sociale de ses porteurs (Fischler, 2007), il n'en reste pas moins qu'on a essayé de mettre en lumière ici certains des mécanismes à travers lesquels le principe de formation des préférences alimentaires réside partiellement dans les stratégies distinctives produites dans les rapports de classe.

\section{BIBLIOGRAPHIE}

BONNET Estelle, «Les critiques gastronomiques : quelques caractéristiques d'une activité experte », Sociétés contemporaines, n 53, 2004, pp. 135-155.

BOURDIEU Pierre, La distinction. Critique sociale du jugement, Paris, Minuit, 1979.

DESLAURIERS Jean-Pierre et KÉRISIT Michèle, « Le devis de recherche qualitative », in Jean POUPART, Jean-Pierre DESLAURIERS, Lionel GROULX, Anne LAPERRIÈRRE et Robert MAYER (eds.), La recherche qualitative : enjeux épistémologiques et méthodologiques, Québec, Gaëtan Morin Éditeur, 1997.

DÓRIA Carlos Alberto, A formação da culinária brasileira, São Paulo, Publifolha, 2009.

ÉRASME de ROTTERDAM Didier, La civilité puérile, Paris, Isidore Liseux Editeur, 1877 (1530) cité in Norbert ELIAS, La Civilisation des Mours, Paris, Calman-Lévy, 1973.

FISCHLER Claude. L'homnivore : le goût, la cuisine et le corps, Paris, Odile Jacob, 1990.

GARCIA-GARZA Domingo, «Prácticas alimenticias y clasificación social. ¿ Los tacos son un alimento 'popular' ", Civitas, vol. 10, n³, 2010, pp. 430-449.

GAULEJAC (de) Vincent, La névrose de classe : trajectoire sociale et conflits d'identité, Paris, Hommes et groupes Editeurs, 1987.

GOFFMAN Erving, La mise en scène de la vie quotidienne, t. 1. La présentation de soi, Paris, Minuit, 1973 (1959).

HOGGART Richard, La culture du pauvre : étude sur le style de vie des classes populaires en Angleterre, Paris, Minuit, 1970 (1957).

LE WITA Béatrix, Ni vue, ni connue. Approche ethnographique de la culture bourgeoise, Paris, MSH, 1988. 
MEDEIROS Marcelo, o que faz os ricos ricos : o outro lado da desigualdade brasileira, São Paulo, Hucitec/Anpocs, 2005.

PEREIRA Daniela Scridelli, Em busca do refinamento : um estudo antropológico da prática da etiqueta, São Paulo, Annablume, 2006.

PINÇON Michel et PINÇON-CHARLOT Monique, Sociologie de la bourgeoisie, Paris, La Découverte, coll. « Repères », 2000.

POULAIN Jean-Pierre, Sociologie de l'alimentation : les mangeurs et l'espace social alimentaire, Paris, PUF, 2002.

PULICI Carolina M., O charme (in)discreto do gosto burguês paulista : estudo sociológico da distinção social em São Paulo, thèse pour le doctorat de sociologie, Université de São Paulo, 2010.

RÉGNIER Faustine, LHUISSIER Anne et GOJARD Séverine, Sociologie de l'alimentation, Paris, La Découverte, coll. « Repères », 2006.

THOMPSON Edward P., La formation de la classe ouvrière anglaise, Paris, Gallimard/Le Seuil, 1988.

WEBER Max, «A distribuição do poder dentro da comunidade. Classes, estamentos e partidos ", in Max WEBER, Economia e Sociedade : Fundamentos de Sociologia Compreensiva, t. 2, Brasília, UNB, 2000.

\section{Journaux, publications de la presse généraliste et critiques gastronomiques}

ALONSO Maria Rita, « Experts em receber », Veja São Paulo, 25 juin 2003. Disponible en ligne: http://veja.abril.com.br/vejasp/250603/sociedade.html [mars 2007].

Anonyme, « Nunca pagaria R\$7.500 num vinho », Folha de São Paulo, 11 septembre 2008, p. E2.

Anonyme, «Folha é o jornal mais lido por jornalistas », Folha de São Paulo, 09 octobre 2008, p. A10.

BASTOS Giuliana, « Banquete para poucos », Revista da Folha, 19 octobre 2008.

BERGAMASCO Daniel, « Jardim Europa deveria ser demolido e virar parque », Folha de São Paulo, 08 juin 2007, p. C6.

BORGES Priscilla, « Taxa de obesidade bate recorde no país », 27 juillet 2012. Disponible en ligne: http://saude.ig.com.br/minhasaude/taxa-de-obesidade-bate-recorde-no-pais/ n1597737564864.html.

FERRARI Márcio, « Letras maiúsculas », Valor econômico, n 509, 23, 24 et 25 juillet 2010, année 11, pp. 4-11.

HORTA Nina, « A carregadora de livros », Folha de São Paulo, 20 novembre 2008, pp. E5.

MOTA Vinícius, « Leitor tem renda e escolaridade altas », in Folha de São Paulo 80 anos, 24 février 2012. Disponible en ligne :

http://www1.folha.uol.com.br/folha/80anos/quem_e_o_leitor.shtml .

NUNES Augusto, « Suave é a vida nos Jardins », Veja SP, avril 2005. Disponible en ligne: http:// vejasaopaulo.abril.com.br/especiais/20anos_jardins1.shtml .

PIEMONTE Marianne et XAVIER Karime, « Nem precisa beber », Revista da Folha, 22 avril 2007, p. 9.

SAITO Bruno, « Feminices », Folha de São Paulo, 05 juin 2005, p. E1. 
YARAK Aretha et ROSA Guilherme, « Cirurgias plásticas : começou a temporada de busca pela perfeição ", Revista Veja, 14 mai 2012. Disponible en ligne: http://veja.abril.com.br/noticia/saude/ comecou-a-temporada-de-cirurgias-plasticas [31/07/2012].

\section{Manuels d'étiquette}

KALIL Gloria, Alô, chics! São Paulo, Ediouro, 2007.

LEÃO Danuza, Na sala com Danuza, São Paulo, Companhia das Letras, 2007.

LEÃO Célia, «Como se comportar » in Jaime PINSKY (ed.), Cultura e elegância : o que se deve fazer e o que é preciso conhecer para ser uma pessoa culta e elegante, São Paulo, Contexto, 2005.

MATARAZZO Claudia, Amante élégante : um guia de etiqueta a dois, São Paulo, Melhoramentos, 2001.

\section{NOTES}

1. Sur le processus à travers lequel certains plats d'origine populaire s'embourgeoisent et, par la médiation de la patrimonialisation et de l'internationalisation, atteignent le statut de "plat national », voir Garcia-Garza (2010).

2. Notre recherche avec les élites de São Paulo tend à conforter cette hypothèse car seulement trois enquêtés (sur trente) ont choisi la feijoada comme l'un de leurs plats préférés. Dans une liste des dix restaurants les plus distingués de la ville, cette spécialité faite à partir d'haricots noirs et d'un mélange de viandes ne figure que dans le menu d'un seul.

3. Sur l'emploi du terme " échantillon » dans la recherche qualitative, voir Deslauriers et Kérisit (1997 : 118).

4. Journaliste ayant un éditorial dans l'un des quotidiens au plus grand tirage [la Folha de São Paulo] et auteur d'ouvrages dans le domaine de la conduite et du savoir-vivre, Danuza Leão lance en 1992 son manuel d'étiquette le plus célèbre, « Na sala com Danuza » [" Au salon avec Danuza »], dont nous discutons ici. Il est resté en tête de la liste des livres les plus vendus pendant un an et a été réédité dans les années 2000 par la Companhia das Letras, qui a été désignée comme la meilleure maison d'édition du Brésil en 2010 (Ferrari, $2010: 4-11$ ).

5. Si cet épisode s'est passé dans un resort, lieu de villégiature inaccessible aux catégories les plus populaires, c'est qu'il n'y a pas une relation mécanique entre les niveaux de revenu et les manières de se comporter.

6. Née dans une famille traditionnelle de São Paulo et ancienne élève du lycée Des Oiseaux, « lieu exclusif »/ " ghetto » des jeunes filles des élites paulistes, Glória Kalil est l'une des plus grandes expertes en matière d'étiquette contemporaine au Brésil. Elle a écrit plusieurs ouvrages dans le domaine de la conduite de soi et travaille comme journaliste et consultrice de mode et de comportement dans la plus grande chaîne de TV du pays [la Rede Globo], diffusant ses conseils à travers des conférences dans des entreprises, des émissions dans des stations de radio et des rubriques journalistiques dans la presse écrite et numérique.

7. Présenté par une "grande dame » issue des élites les plus anciennes de São Paulo et épouse d'un important industriel brésilien, ce manuel rassemble des textes de quelques-uns des plus importants agents de l'establishment culturel local (comme des professeurs de grandes écoles et des critiques culturels des journaux les plus importants).

8. Ce n'est pas un hasard, ainsi, si les chaînes de télévision ne proposent qu'aux femmes favorisées des émissions propices à l'accumulation des savoirs "gratuits ». Diffusant les résultats d'une enquête sur la consommation, une critique culturelle sur le profil des programmes adressés aux femmes (Saito, 2005 : E1) révèle qu'alors que les chaînes payantes - dont les 
téléspectateurs ont une formation scolaire supérieure - discutent de l'histoire de la gastronomie, faisant des programmes sur la cuisine un prétexte pour se cultiver, la chaîne publique (nonpayante) n'approche la nourriture que comme satisfaction de besoins primaires (divulgation de recettes et préparation de plats).

9. Si les différentes visions médiatiques n'ont pas toutes le même poids dans la construction des représentations sociales, il est possible de dire que la Folha de São Paulo, en tant que quotidien national ayant le plus grand tirage et étant le plus lu par les journalistes du pays (sauf ceux de Rio), est certainement un des véhicules les plus puissants dans la création des effets de réalité. Selon un sondage de l'année 2000 appliqué à 267 personnes, son lecteur typique appartient aux tranches d'éducation et de revenu les plus élevées (Anonyme, 2008 : A10; Mota, 2012).

10. L'esthétisation de la cuisine, qui mène à déplacer l'intérêt de la «matière " vers la «manière » (de présenter, de servir et de jouir des aliments), se donne bien à voir aussi dans la critique "Même pas besoin de boire ", dédiée à l'exposition des boissons qui «font partie du répertoire du bon buveur ». Ayant présenté la bouteille de porcelaine anglaise du whisky Royal Salute 21, la Vodka Wyborowa Single Estate, au flacon dessiné par Frank Gehry, et le vin Villa Francioni Rosé, conservé dans une bouteille française avec un bouchon de liège portugais et des capsules d'étain espagnoles, on suggère que des boissons "avec une silhouette splendide, un blend unique et un parfum impérissable " peuvent même ne pas être dégustées car « contenu mis à part, elles font soupirer » (Piemonte et Xavier, $2007: 9$ ).

11. Étudiant les cours d'étiquette à São Paulo dans les années 1990, l'anthropologue Daniela Scridelli (2006) observe que les professeurs qui ont hérité de ce type de comportement se présentent comme les détenteurs légitimes des «bonnes manières ", au détriment de ceux qui les ont apprises ultérieurement, en dehors d'un cadre familial.

12. La plus grande autonomie de certaines pratiques par rapport à l'action de l'école reproduit les principes de hiérarchisation structurant le champ de production de ces pratiques. Prenons le champ des critiques gastronomiques qui, moins professionnalisé que celui de la critique artistique, est formé par des agents dont l'expertise ne découle pas de la possession d'un diplôme et émerge comme jugement semi-savant et autorisé (Bonnet, 2004).

13. Issue d'une des principales familles de l'élite italienne de São Paulo, Claudia Matarazzo est l'auteure de plusieurs manuels d'étiquette et occupe le poste de chef du protocole dans le gouvernement de l'État de São Paulo.

14. La cuisine japonaise est aussi de plus en plus présente dans le circuit de la haute gastronomie pauliste.

15. Selon Dória (2009), l'approche ethnique de l'alimentation au Brésil - qui se manifeste dans l'expression « la cuisine brésilienne » - ne retient pas assez le fait que les Indiens, les esclaves africains et les Portugais n'ont pas eu le même pouvoir de légitimer leurs habitudes alimentaires respectives. En plus, elle simplifie énormément la diversité culinaire de ce pays aux dimensions continentales. Cette vision stéréotypée serait, donc, plus rhétorique qu'empirique, servant surtout à l'industrie du tourisme. Ainsi, un plat brésilien typique et très diffusé comme l'escalope de veau parmigiana - qui n'existerait pas à Parme d'ailleurs - n'est pas reconnu comme coisa nossa parce qu'il ne correspond pas à cette approche ethnique. D'après l'auteur, la cuisine cosmopolite de la ville de São Paulo (qui exprime l'origine multiple de ses habitants) constitue le lieu par excellence des tendances contemporaines de la cuisine brésilienne.

16. Ce poisson encore perçu comme un aliment d'exception est devenu un symbole de «la médicalisation de l'alimentation quotidienne » (Poulain, 2002). Dans un marché en plein air qui se tient tous les samedis dans un quartier «bobo ", un humble vendeur dit à son collègue: « Voilà le poisson que les médecins nous disent de manger ».

17. À la question "croyez-vous que c'est bien de manger et de boire à volonté ?", tous les informateurs, aussi bien les hommes que les femmes, ont répondu négativement, à l'exception d'un seul. 
18. Même si notre approche n'a pas conduit à repérer d'importantes différences de genre en matière d'attention portée au contrôle du poids, on peut faire l'hypothèse que le souci pour le corps est plus fort parmi les femmes, comme le soutient Fischler (1990), car au Brésil, deuxième pays (après les États-Unis) le plus important en matière de chirurgie plastique, les hommes ne représentent, en 2008, que 12\% du total de ces interventions (Yarak et Rosa, 2012).

19. Le couscous brésilien (et surtout le pauliste) n'a pas grand-chose à voir avec celui qui est originaire du Maghreb. Fait à partir de la farine de maïs et nettement plus mouillé, il est fourré de sardines, de crevettes et de cœurs de palmier et contient des légumes (comme la tomate) mélangés aux oignons et aux aulx préalablement sautés.

20. La dernière étude faite par le ministère de la santé révèle que $15,8 \%$ des Brésiliens sont obèses (environ 30 millions de personnes), un niveau record dans l'histoire du pays. Selon cette recherche, plus de la moitié (52\%) de la population ayant moins de huit ans d'études est en surpoids; parmi ceux qui ont plus de douze ans de scolarité le taux est de $47 \%$. Le découpage selon le sexe montre que pour les femmes les taux de surpoids et d'obésité sont de $35 \%$ et de $11 \%$ parmi celles qui ont le plus d'années d'études, alors que pour celles qui ont moins fréquenté l'école les pourcentages montent à $52 \%$ et $20 \%$ respectivement. Le niveau d'études a un effet inverse dans le cas des hommes: parmi ceux qui ont fréquenté l'école plus de 12 ans, $60 \%$ sont en surpoids et $17 \%$ sont obèses, tandis que les taux sont de $51 \%$ et de $16 \%$ pour ceux qui ont le niveau d'études le plus bas (Borges, 2012).

21. L'investissement esthétique des élites dans la vie de tous les jours se donnerait à voir de façon beaucoup plus claire en mettant l'alimentation en rapport avec toutes leurs autres pratiques culturelles, ce que nous avons fait ailleurs (Pulici, 2010), mais ne pouvons pas faire dans le cadre de cet article.

22. Cette informatrice raconte qu'elle se dispute avec sa fille quand cette dernière ne tient pas à avoir une table bien présentée dans les occasions où elles font appel au Arábia - le restaurant le plus distingué de la ville en matière de cuisine libanaise -, qui livre des repas à domicile. Vu que cette enquêtée a environ 60 ans et sa fille, qui habite en Europe, 30, on peut faire l'hypothèse que, comme le fait remarquer Domingo Garcia-Garza (2010), les jeunes sont les plus inclinés à s'affranchir de conventions concernant l'étiquette à table.

23. L'élargissement d'une culture gastronomique semble faire partie de ses efforts de perfectionnement culturel : au cours de l'entretien, il nous parle avec de la joie d'un cours de cuisine fait à Milan et d'un autre cours de cuisine thaillandaise suivi à New York. Il reçoit souvent autour de trente personnes pour déguster de la paella, une de ses spécialités.

\section{RÉSUMÉS}

Les enjeux de distinction liés à l'alimentation ont été un thème cher aux chercheurs de sciences sociales, et sont précisément au centre de la discussion de cet article qui abordera les élites brésiliennes à partir de leurs pratiques et de leurs jugements concernant l'univers de la gastronomie. Sur la base de trente entretiens semi-directifs avec des représentants des classes supérieures de São Paulo, des manuels de savoir-vivre récemment publiés au Brésil et des critiques gastronomiques parues dans la presse pauliste entre 2005 et 2009, nous nous attacherons, dans un premier temps, à décrire certaines normes qui s'imposent aujourd'hui comme légitimes en matière d'alimentation et de manières de table. Dans un deuxième temps, nous nous centrerons sur la façon dont des groupes aisés paulistes réagissent aux appels 
d'esthétisation de la cuisine et sur les représentations que leurs membres construisent d'euxmêmes et des autres à partir de leurs attitudes alimentaires, lesquelles contribuent aussi à former la « réalité » de ces élites.

Distinction issues related to food consumption have been a major theme in the field of social sciences and are precisely at the centre of this article which will approach the Brazilian elites from the point of view of their practices and judgments concerning the realm of gastronomy. Based on semi-structured interviews with São Paulo's elites, etiquette books recently published in Brazil, and gastronomic critiques in the press between 2005 and 2009, this article begins by focusing on the description of some norms that are nowadays legitimated in terms of cuisine and table manners. In a second step, we will concentrate on how the fractions of the upper classes of São Paulo (Brazil's economic capital) respond to these appeals for food aestheticization, as well as the representations that their own members build of themselves and others based on their eating attitudes, a process that also contributes to shaping the « reality» of these social group.

Los aspectos más importantes sobre la alimentación y la distinción social ha sido bastante estudiados por las ciencias sociales, y constituye el tema central del presente artículo. Abordaremos las practicas y los juicios sobre el universo de la gastronomía de las élites brasileñas. Partiendo de una base de treinta entrevistas semi-estructurada con representantes de las clases altas de São Paulo, de manuales de comportamiento recientemente difundidos en Brasil y de críticas gastronómicas publicadas en la prensa paulista entre los años 2005 y 2009, nos concentraremos, en primer lugar, en la descripción de ciertas normas que actualmente se impusieron como legítimas en materia de alimentación y de modales en la mesa. En segundo lugar, nos centraremos en el modo en que algunas fracciones de las clases acomodadas de la capital económica del país reaccionan a los llamados de la estetización de la cocina, y en las representaciones que sus miembros construyen de ellos mismos y de otros a partir de sus hábitos alimentarios, lo que contribuye al mismo tiempo a conformar la « realidad » de estas élites.

INDEX

Palabras claves : discurso gastronómico, estetización de la cocina, dominio del cuerpo, distinción social, élites, Brasil

Mots-clés : discours gastronomiques, esthétisation de la cuisine, maîtrise du corps, distinction sociale, élites, Brésil

Keywords : gastronomic discourses, aestheticization of food, body control, social distinction, elites, Brazil

\section{AUTEUR}

\section{CAROLINA PULICI}

Carolina Pulici est maître de conférences en sociologie à l'UNIFESP (Université Fédérale de São Paulo). La préparation de cet article a bénéficié du soutien financier d'une bourse de postdoctorat de la FAPESP (Fundação de Amparo à Pesquisa do Estado de São Paulo). Email : carolinapointpuliciatunifesppointbr. 